\title{
Dwarf Galaxy Age-Metallicity Relationships
}

\author{
Evan D. Skillman \\ Astronomy Department, University of Minnesota, Minneapolis, MN 55123, USA
}

Abstract. I present a brief review of current work on dwarf galaxy age-metallicity relationships.

Keywords. galaxies: abundances, dwarf, evolution, Local Group

\section{Observing Age-Metallicity Relationships}

In principle, deriving age-metallicity relationships for dwarf galaxies should be easy. Step one: Measure the ages of the stars. Step two: measure the metallicities of those stars. Step three: plot the results (including error bars).

In practice, there are some complications. For measuring the ages of stars, it is best to use populous star clusters. Unfortunately, the ideal case of a uniform distribution of star clusters in time and galactic radius is very far from the typical distribution. This means that we will need to work with field stars.

Measuring the metallicities of the stars also has its difficulties. It is best to use high dispersion spectra to measure the chemical abundances of a star. Given the distances of even the closest galaxies, in most cases this is not possible. Ca triplet observations can be used for some nearby galaxies, but for most galaxies we will need to work with the color magnitude diagram and fight with the age-metallicity degeneracy.

Given the above, clearly, the constraints on the age-metallicity relationships will be loose, at best. There are some simple tests to check whether the current results are on the right track. For example, one expects the metallicity to be rising monotonically. Also, one expects that the chemical enrichment should roughly follow the star formation history. If these simple tests are met (without being assumed as priors) then one gains confidence that derived age-metallicity relationships can provide useful constraints on our ideas about galaxy evolution.

\section{Calibrations}

One can consider our Milky Way and the Magellanic Clouds as calibrators for techniques which are used to derive age-metallicity relationships. As a note of caution, it is interesting to look at the comparison presented in Chiappini et al. (1997) of recently derived age-metallicity relationships for our Milky Way. The fact that this can give rise to some controversy tells us that we must be conservative in our approach.

I find the results from Cole et al. (2005) very intriguing. They have derived the agemetallicity relationship for the LMC bar from spectroscopic observations (Figure 1 left). Considering the sizes of the time bins and the error bars in metallicity, such a plot provides strong constraints on the evolution of this galaxy.

Figure 1 (right) shows the color magnitude diagram for the red giant stars observed in the LMC bar. Spectroscopic metallicities have been obtained for many of these stars, and are highlighted in the figure. Overall, one sees a trend of low metallicity stars to the left and high metallicity stars to the right - which recovers the expected trend that more metal poor stars have bluer colors. However, the intermediate metallicity stars cover 

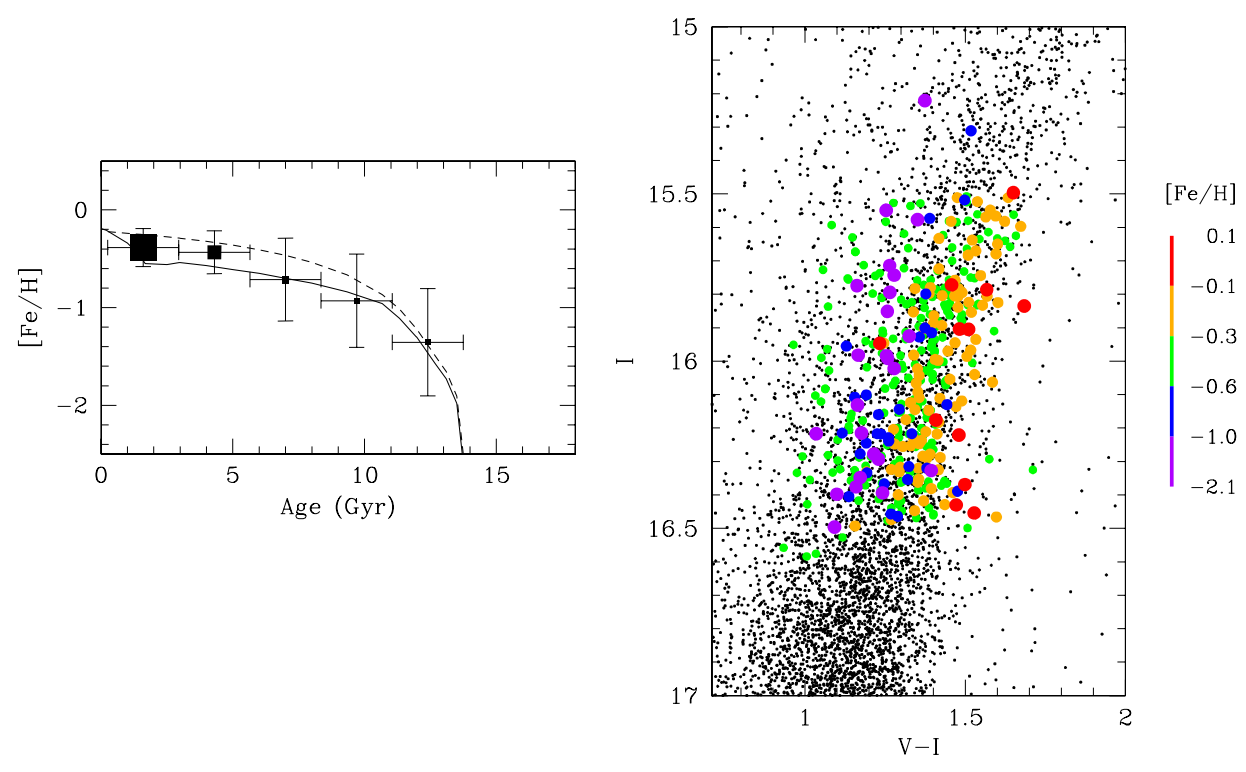

Figure 1. (left) Age-metallicity relation for a field in the the LMC bar from Cole et al. (2005), binned into 2.7 Gyr intervals from $0.2-13.7 \mathrm{Gyr}$. The symbol size is proportional to the number of stars in each age bin. Error bars show the bin width and the rms dispersion of abundances in each bin. Chemical evolution models from Pagel \& Tautvaišienè have been overplotted, for both bursting (solid line) and continuous (dashed line) star-formation histories. (right) Color magnitude diagram of the red giant branch star in the LMC bar field from Cole et al. (2005); the stars with spectroscopic measurements are coded according to their metallicity (see original article). For the extreme metal-poor and metal-rich stars the RGB color correlates with metallicity, but stars near the peak of the metallicity distribution function are scattered across the entire width of the RGB.

almost the full range in observed colors, and this is probably the effect of different ages spreading the stars in color.

\section{Dwarf Spheroidals}

The Milky Way dwarf spheroidal companions are close enough that one can obtain abundances from color-magnitude diagrams or from actual spectroscopic measurements. A nice comparison of age-metallicity diagrams drawn from HST WFPC2 imaging is presented in Dolphin (2002). Here one sees that dSphs which had most of their star formation early on, show little evidence of significant chemical evolution at later time (e.g., Leo II). On the other hand, dSph galaxies which show evidence of significant star formation right up to very recent times show metallicity increasing with continued star formation (e.g., Leo I).

When spectra are obtained of individual stars, the derived metallicities enforce the picture produced by the CMD studies. Tolstoy et al. (2003) compare high dispersion spectroscopic abundances and Ca triplet metallicities for stars in several Milky Way dSph companions. While the Ca triplet observations yield relatively large numbers of stars and support the CMD studies, the high resolution studies provide actual element abundances and elemental abundance ratios (e.g., Shetrone et al. 2003), which provide a whole new set of constraints. It is possible to add chronometers from relative abundances and even test for departures from a universal IMF. It is also possible to derive radial gradients 
in abundances. Finally, the spectroscopic observations provide kinematic information, which, to date, has produced some surprises. In the case of the Sculptor dSph, two kinematically distinct populations are detected (Tolstoy et al. 2004). Two large VLT programs (PIs = Tolstoy, Gilmore) promise to bring a wealth of data in the very near future.

\section{Dwarf Irregulars}

The dwarf irregular galaxies present a greater challenge simply because they are found at larger distances. If the desired goal is to produce a global view of the evolution of a galaxy, then the individual star photometry must reach below the oldest age main sequence turn-off. Skillman et al. (2003) published the deepest color-magnitude diagram of an isolated dwarf irregular galaxy (IC 1613). The surprising result was a predominance of intermediate age star formation (between the ages of 3 and $6 \mathrm{Gyr}$ ). The inferred star formation history for IC 1613 looks very similar to that of the Milky Way companion dSph Leo I.

Real advances on dwarf irregular galaxies will be coming in the near future. In cycle 14, two programs (PIs = Gallart, Cole) were allocated deep ACS imaging of the isolated Local Group galaxies Leo A, IC 1613, and LGS-3 (Pisces). The resultant color magnitude diagrams will reveal the total star formation histories for these galaxies. The chemical evolution of these galaxies will be further constrained by spectroscopic measurements of their brightest red giant branch stars.

\section{Chemical Evolution of Dwarfs}

The biggest problem for interpretation is the history of the gas content in dwarf galaxies. We have no way to observe this directly. We can assume that the star formation rate is equal to the infall rate, but assuming the answer is less than satisfactory. Chemical abundance ratios may provide clues concerning the timescales of gas history, if not the gas history itself. For isolated dwarfs, it may be that the simple closed box model is not a terrible approximation. Does one expect very much late time infall for low mass, isolated systems? For higher density environments, the situation is complicated by interactions and stripping. Adding in the uncertainties in the yields and the possibility of IMF variations, there remains much work to be done on the observational side.

\section{Acknowledgements}

I would like to acknowledge my collaborators, too numerous to mention here, that have allowed me to participate in this work. I would also like to thank the conference organizers for inviting me to participate in this very lively conference. My recent work in this field has been supported by NASA LTSARP grant No. NAG5-9221, numerous NASA HST grants including HST-GO-7496, HST-GO-10505, and HST-GO-10590, and the University of Minnesota.

\section{References}

Chiappini, C., Matteucci, F., \& Gratton, R. 1997, ApJ, 477, 765

Cid Fernandes, R., Mateus, A., Sodré, L., Stasińska, G., \& Gomes, J. M. 2005, MNRAS, 358, 363

Cole, A. A., Tolstoy, E., Gallagher, J. S., III, \& Smecker-Hane, T. A. 2005, AJ, 129, 1465

Dolphin, A. E. 2002, MNRAS, 332, 91 
Pagel, B.E.J., \& Tautvaišienè, G. 1998, MNRAS, 299, 535

Shetrone, M., Venn, K. A., Tolstoy, E., Primas, F., Hill, V., \& Kaufer, A. 2003, AJ, 125, 684

Skillman, E. D., Tolstoy, E., Cole, A. A., Dolphin, A. E., Saha, A., Gallagher, J. S., DohmPalmer, R. C., \& Mateo, M. 2003, ApJ, 596, 253

Tolstoy, E., Venn, K. A., Shetrone, M., Primas, F., Hill, V., Kaufer, A., \& Szeifert, T. 2003, AJ, 125,707

Tolstoy, E., et al. 2004, ApJL, 617, L119

\section{Discussion}

Roberto Cid Fernandes: A comment and a question: The comment is that poster no. 83 show that $\mathrm{Z}$ (star) can be recovered from synthesis of integrated spectra. The question: chemical build-up time scales should change from small low mass to massive galaxies. Do you see a sign of that among your dwarf galaxies?

SkILlman: Concerning your comment, I think it may now be possible to acquire integrated spectra of the nearby galaxies for which we are obtaining very detailed star formation histories. By comparing the results of these two independent analyses, we can gain confidence in both techniques. Regarding your question, the large variety of star formation histories observed in the nearby dwarf galaxies indicate that there is not a simple answer to your question. Environmental influences must play a large role in determining the star formation history of a dwarf galaxy, so that looking for a signature as a function of mass only becomes a challenging proposition.

LAURA PORTINARI: A general comment on the AMR: it grows fast at early times and then appears to level off mostly because $[\mathrm{Fe} / \mathrm{H}]$, and other typical metallicity indicators, are logarithmic. A plot of $\mathrm{Z}$ (mass fraction of heavy elements) vs. age is much closer to linear.

SKILlman: (a better answer than the one I gave in Prague) - You make a very important point. Although the logarithmic metallicity is often plotted (best for certain types of comparisons - especially since the input stellar evolution models are calculated on a logarithmic grid), for the purpose of comparing age-metallicity relationships it is very valuable to plot the metallicity in linear units. In this way, the chemical enrichment can be directly compared to the star formation rate and it is easier to judge whether the two are in good agreement.

ANONYMOUS: If things are so complex for resolved populations, what hope is there for interpreting properties at $\mathrm{z} \sim 1$ and beyond? Could you comment?

SKILLMAN: In the nearest galaxies we are pushing for very detailed histories of galaxies with high time resolution and small error bars. In conducting these exercises I am very impressed with the quality of the stellar evolution models which form the backbone for all of this work. This high quality yields the potential for powerful diagnostics. Clearly things will be more difficult at higher redshift, but one should not be discouraged, as your question might imply. Quoting from Cid-Fernandes et al. (2005), "We find that spectral synthesis provides reliable physical parameters as long as one does not attempt a very detailed description of the star formation and chemical histories." I think this makes a lot of sense. 